\title{
The efficacy of DNA vaccination is enhanced in mice by targeting the encoded protein to dendritic cells
}

\author{
Godwin Nchinda, ${ }^{1,2}$ Janelle Kuroiwa, ${ }^{1}$ Margarita Oks, ${ }^{1}$ Christine Trumpfheller, ${ }^{1}$ \\ Chae Gyu Park, ${ }^{1}$ Yaoxing Huang, ${ }^{3}$ Drew Hannaman, ${ }^{4}$ Sarah J. Schlesinger, ${ }^{1,3}$ \\ Olga Mizenina, ${ }^{1}$ Michel C. Nussenzweig, ${ }^{5}$ Klaus Überla, ${ }^{2}$ and Ralph M. Steinman ${ }^{1}$
}

\begin{abstract}
1 Laboratory of Cellular Physiology and Immunology, The Rockefeller University, New York, New York, USA. 2Department of Molecular and Medical Virology, Ruhr-University Bochum, Bochum, Germany. ${ }^{3}$ The Aaron Diamond AIDS Research Center, New York, New York, USA. ${ }^{4}$ Ichor Medical Systems, San Diego, California, USA. ${ }^{5}$ Laboratory of Molecular Immunology, Chris Browne Center for Immunology, The Rockefeller University, New York, New York, USA.
\end{abstract}

\begin{abstract}
DNA vaccines promote an immune response by providing antigen-encoding DNA to the recipient, but the efficacy of such vaccines needs improving. Many approaches have considerable potential but currently induce relatively weak immune responses despite multiple high doses of DNA vaccine. Here, we asked whether targeting vaccine antigens to DCs would increase the immunity and protection that result from DNA vaccines. To determine this, we generated a DNA vaccine encoding a fusion protein comprised of the vaccine antigen and a single-chain $\mathrm{Fv}$ antibody ( $\mathrm{scFv}$ ) specific for the DC-restricted antigen-uptake receptor DEC205. Following vaccination of mice, the vaccine antigen was expressed selectively by DCs, which were required for the increased efficacy of MHC class I and MHC class II antigen presentation relative to a control scFv DNA vaccine. In addition, a DNA vaccine encoding an HIV gag $\mathrm{p} 41$-scFv DEC205 fusion protein induced 10-fold higher antibody levels and increased numbers of IFN- $\gamma$-producing $\mathrm{CD4}^{+}$and $\mathrm{CD8}^{+} \mathrm{T}$ cells. After a single i.m. injection of the DNA vaccine encoding an HIV gag $\mathrm{p} 41-\mathrm{scFv}$ DEC205 fusion protein, mice were protected from an airway challenge with a recombinant vaccinia virus expressing the HIV gag $\mathrm{p} 41$, even with $1 \%$ of the dose of nontargeted DNA vaccine. The efficacy of DNA vaccines therefore may be enhanced by inclusion of sequences such as single-chain antibodies to target the antigen to DCs.
\end{abstract}

\section{Introduction}

DNA vaccination is at the forefront of efforts aimed at developing vaccines against challenging infectious diseases, including HIV AIDS as well as emerging strains of influenza and SARS (reviewed in refs. 1-4). Innovations in vaccine design have improved the immunogenicity of DNA vaccines, leading to licensure of 2 candidate DNA vaccines for nonhuman applications (reviewed in refs. $5,6)$. However, in humans and experimental animals, repeated and high doses of DNA must be injected.

A fundamental initial step in vaccination is that the vaccine proteins need to be taken up, processed, and presented by DCs, which are antigen-presenting cells that initiate and control many aspects of the immune response. Following intradermal or i.m. injection of a plasmid DNA vaccine in mice, the encoded gene is expressed in transfected keratinocytes and myocytes at the site of inoculation (7) as well as a small number of DCs (8-12). Keratinocytes and myocytes lack MHC class II and costimulatory molecules, which renders them poorly effective at presenting antigen and priming naive immune cells (reviewed in ref. 13), and they do not have ready access to T cells in lymphoid tissues, as is the case for DCs $(14,15)$. Therefore, it is thought that immune priming begins through the function of a few transduced DCs and that antigen secreted from

Nonstandard abbreviations used: $\mathrm{CHO}$, Chinese hamster ovary; DT, diphtheria toxin; scDEC, single-chain antibody to murine DC205; scFv, single-chain Fv antibody; TAP, transporter for antigenic peptides.

Conflict of interest: R.M. Steinman and M.C. Nussenzweig have a financial interest in Celldex Therapeutics, which is developing antibody-based vaccines that target dendritic cells.

Citation for this article: J. Clin. Invest. 118:1427-1436 (2008). doi:10.1172/JCI34224. other long-lived transfected cells then boosts immunity (16-23). We hypothesized that the weak immunogenicity of DNA vaccines could be overcome by directly targeting the encoded vaccine protein to DCs, particularly during the later stages of immunization.

Recent studies have demonstrated that antigens can be targeted selectively to DCs in vivo when antigen is incorporated into an antibody against the DC endocytic receptor, DEC205/CD205 (24). This targeting is accompanied by efficient antigen processing and presentation on MHC class I and II products as well as strong and protective $\mathrm{T}$ cell immunity $(25,26)$. Demangel et al. reported on the capacity of a single-chain antibody to murine anti-DEC205 (scDEC) to target DNA vaccine antigens in mice (27). They observed approximately a 2 -fold increase in $\mathrm{B}$ and $\mathrm{T}$ cell responses to a mycobacterial antigen but did not establish whether targeted delivery of vaccine to DCs was taking place or whether the dose of DNA vaccine could be reduced and maintain efficacy. As a result, it is not clear whether the targeted delivery to DCs overcomes some of the key obstacles to current DNA vaccines.

We have evaluated the incorporation of a scDEC antibody into DNA vaccines. We will show that this approach greatly enhances antigen presentation by DCs in lymphoid tissues relative to nontargeted DNA vaccine in mice, and concomitantly, that single and much lower doses of DNA are effective in inducing immunity and protection.

\section{Results}

Construction and expression of scDEC DNA vaccines. To better target DNA vaccines to DCs, a eukaryotic expression plasmid was constructed to encode scDEC fused to a vaccine protein. DEC205 


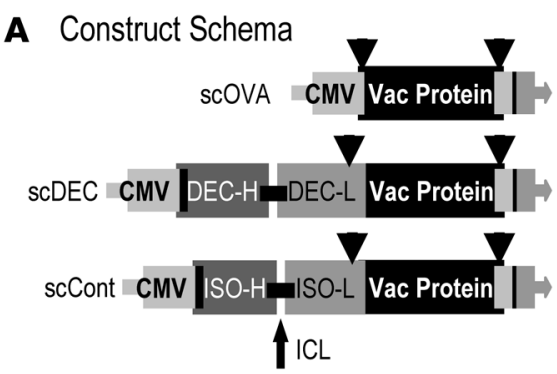

D Ovalbumin

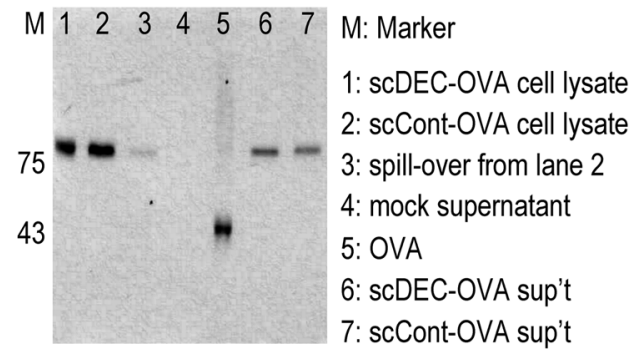

B
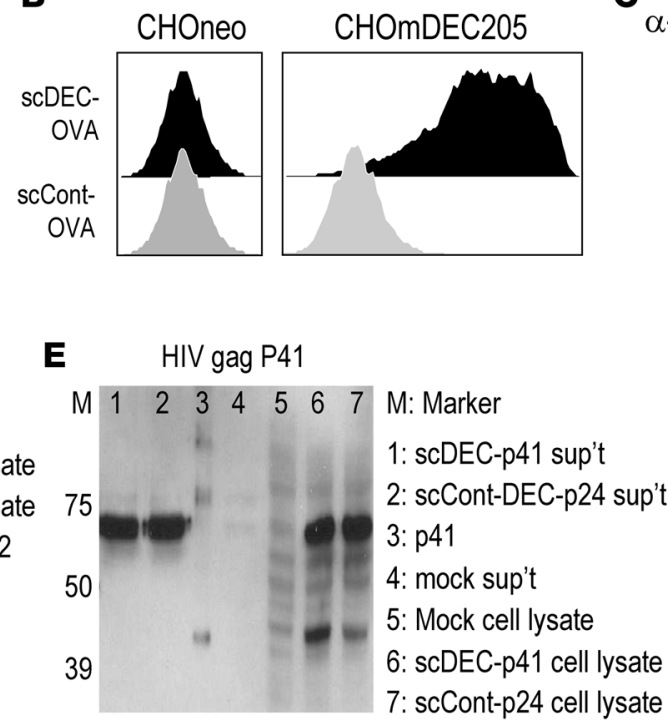

C $\alpha$-DEC-OVA/scDEC-OVA

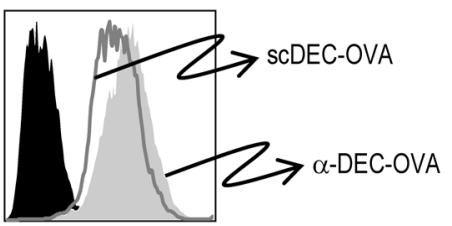

F $\quad$ HIV gag P24

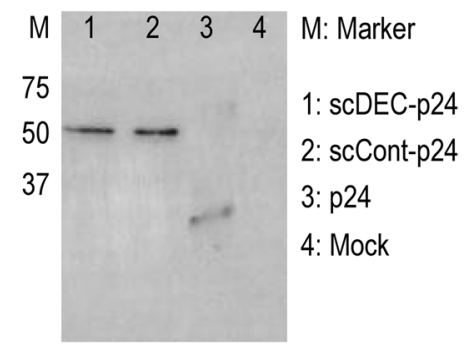

Figure 1

Design and characterization of antigen fused to single-chain antibody to DEC205. (A) Map of expression cassettes encoding vaccine protein (Vac protein) fused to single-chain scDEC and scControl. Heavy-chain (H) and light-chain (L) cDNAs of each mAb were connected by an interchain linker (ICL) and cloned in frame upstream of the cDNA for OVA, HIV gag fragments (p41, p24), or GFP in a eukaryotic expression plasmid containing the human CMV immediate early promoter and a bovine growth hormone polyadenylation signal (pAd). LP, leader peptide; Tag, myc and $6 \times$ histidine tag. (B) Binding to $\mathrm{CHO}$ cells expressing murine DEC205 (CHOmDEC205) or CHOneo control cells of recombinant single-chain mAbs-OVA fusion proteins detected by an OVA-specific, FITC-labeled secondary antibody. (C) Right part shows an overlay of the binding of the parental bivalent antibody (DEC-OVA) compared with scDEC-OVA. (D-F) Supernatants and lysates of 293T cells transfected with expression plasmids for scDEC or scControl conjugated to either OVA, HIV gag p41, HIV gag p24, or empty expression plasmid (pcDNA3.1; Invitrogen) were Western blotted using mAbs against OVA (D) or anti-HIV gag p24 (E and F).

is an antigen uptake receptor on DCs, and the open reading frames for the variable regions of the light and heavy chains of the parental anti-DEC205 antibody were fused to the protein sequence. A control plasmid from a nonreactive antibody was also constructed with different variable light- and heavy-chain $\mathrm{V}$ regions (Figure 1A). cDNAs for OVA, HIV gag p41 (anti-p41) and p24 (anti-p24), and GFP were cloned in frame with the scDEC or the control antibody. To verify that the scDEC protein retained the antigen-binding activity of the parental antibody, we measured specific binding to Chinese hamster ovary ( $\mathrm{CHO}$ ) cells stably expressing murine DEC205 receptor. Only supernatants from scDEC-OVA-transfected 293T cells (Figure 1, B and C) showed binding to DEC205-expressing CHO cells. When we compared the DEC205-binding efficacy of the scDEC-OVA relative to the parental bivalent antibody, both had strong and specific binding ability to DEC-transfected $\mathrm{CHO}$ cells but not to control CHO cells (Figure 1C). We also compared the expression of scDEC and scControl DNA using the GFP reporter and found comparable expression in transiently transfected 293T cells (Supplemental Figure 1, A and B; supplemental material available online with this article; doi:10.1172/JCI34224DS1), but only the scDEC-EGFP vaccine bound selectively to $\mathrm{CHO}$ cells expressing mouse DEC205 (Supplemental Figure 1C). Western blot analysis of supernatants and lysates of 293T cells transfected with expression plasmids for different single-chain antibodies conjugated to the different vaccine proteins was then carried out and revealed comparable expression and secretion of scDEC and scControl fusion proteins of the expected size
(Figure 1, D-F). Therefore scDEC fusion antibodies retain the binding capacity of the parental anti-DEC205 antibody and are secreted by transiently transfected cells.

Improved in vivo targeting of antigen to DCs using scDEC DNA vaccine. To determine whether scDEC DNA vaccines would improve antigen delivery to DCs in vivo, we used scDEC-OVA because sensitive and valuable reagents are available to monitor the successful processing and presentation of OVA on MHC class I and class II in vivo. Graded doses of scDEC- and scControl-OVA DNA were injected i.m. in combination with electroporation. It has previously been shown that electroporation enhances the efficacy of DNA vaccination $(28,29)$. From 4 to 5 days after DNA injection, OVA-specific, TCR transgenic T cells were labeled with CFSE and transferred into the vaccinated mice. If OVA antigen were being presented in vivo, these $T$ cells would proliferate over the ensuing 3 days, yielding half the amount of the CFSE label per cell with each cell division. In the case of ScDEC-OVA, we found that the majority of the TCR transgenic T cells went into cell cycle, i.e., both $\mathrm{CD}^{+}$MHC-I-restricted OT-I cells and CD4 ${ }^{+}$MHC-II-restricted OT-II cells. The OT-I cells responded to the DNA vaccine at all doses, 3-100 $\mu \mathrm{g}$, whereas the OT-II cells only showed proliferation at the higher doses of DNA. This reflects the fact that OT-I cells are unusually efficient in being able to recognize low pM doses of specific OVA peptide. In contrast, a $100-\mu \mathrm{g}$ DNA dose of scControl OVA DNA was required to induce OT-I responses that were comparable to those obtained with $3 \mu \mathrm{g}$ of scDEC-OVA, and no proliferation was observed for $\mathrm{CD}^{+}$OT-II T cells at any dose of scControl OVA DNA (Figure 2A). 
A

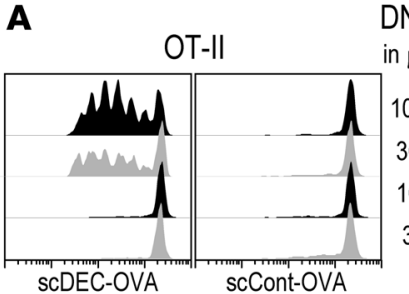

C

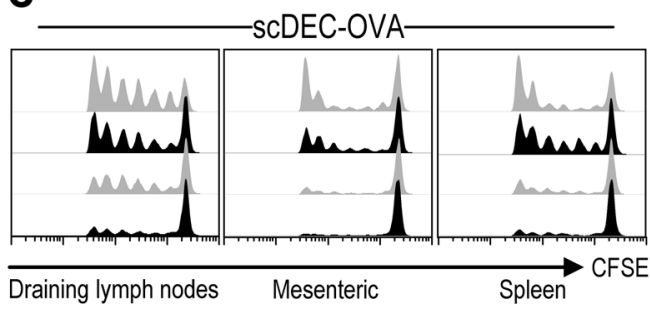

D

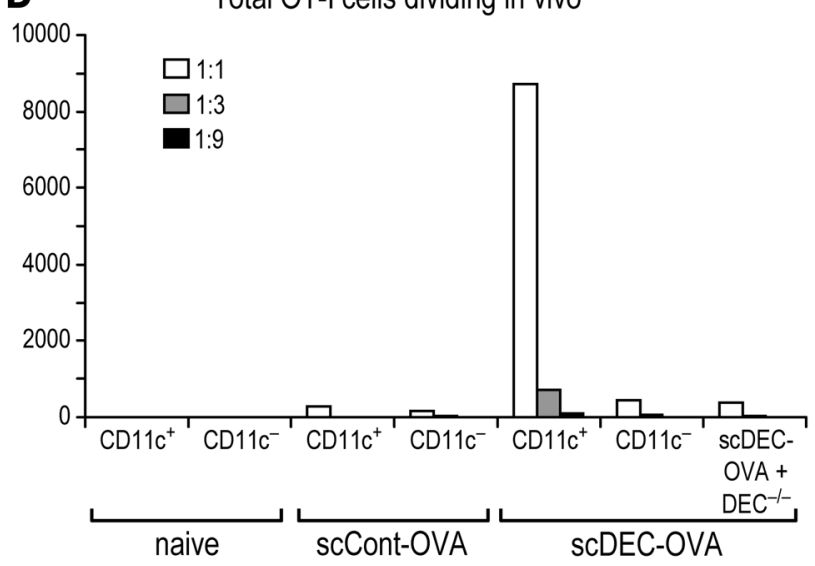

Week 1

Week 2

Week 3

Week 4
B
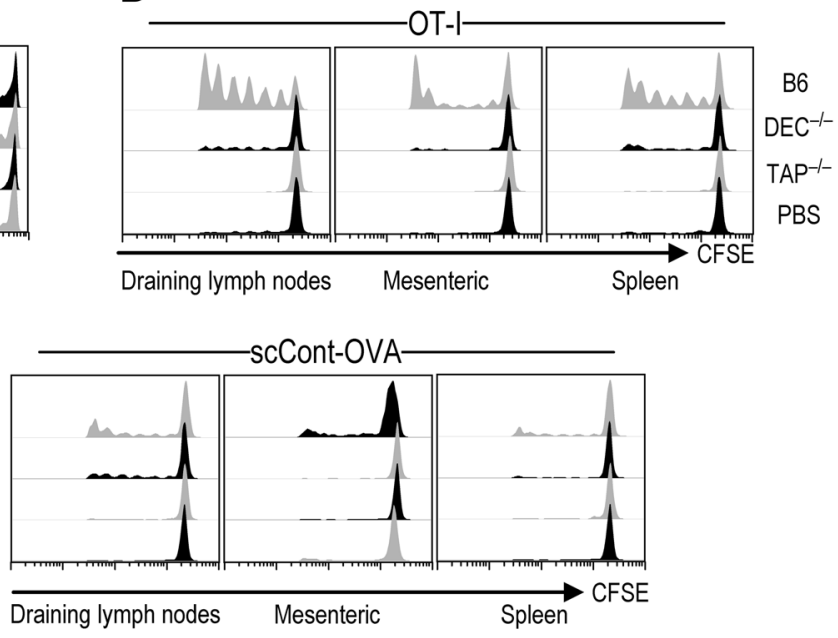

E

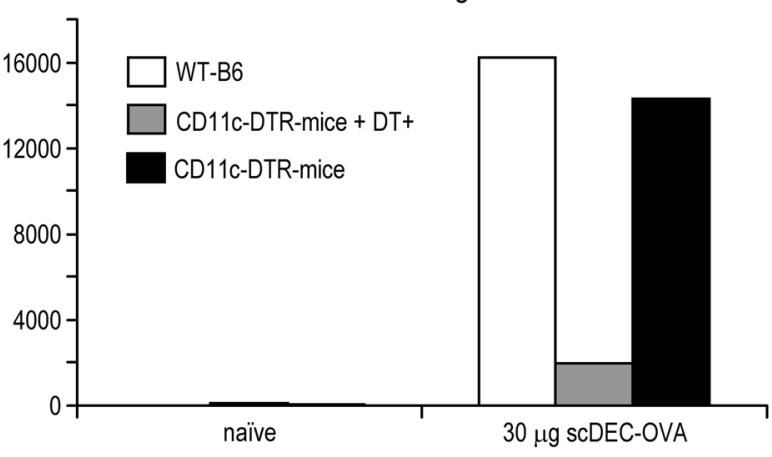

DNA vaccine dose

Figure 2

Antigen presentation to OVA-specific T cells following in vivo targeting of DCs with OVA DNA fused to a single-chain antibody to DEC205. (A) Mice were immunized i.m. with graded doses of DNA, and 4 days later, $2 \times 10^{6}$ CFSE-labeled CD8 ${ }^{+}$OT-I or $3 \times 10^{6}$ CD4+ OT-II TCR transgenic T cells were injected i.v. At day 7 , draining lymph node cells were harvested and cell division was evaluated by flow cytometry in CD4+ OT-II cells with $\mathrm{V}_{2} \mathrm{~T}$ cell receptor (20.1) antibody and in CD8+ OT-I cells with $\mathrm{V} \beta_{5.1 / 52^{+}} \mathrm{T}$ cell receptor (MR9-4) antibody. (A) Control mice received similar amounts of the scControl-OVA DNA vaccine. (B) As in A, but the experiments were performed with DEC ${ }^{-/}$, TAP ${ }^{-/-}$, or WT B6 mice using $30 \mu \mathrm{g}$ of each DNA vaccine. Representative results from 3 experiments are shown. (C) Longevity of in situ antigen presentation after vaccination with $30 \mu \mathrm{g}$ DNA, then evaluation by antigen presentation to $2 \times 10^{6}$ OT-I T cells injected at the indicated time points. (D) scDEC-OVA targets antigens to DCs after DNA vaccination. Mice were immunized with $30 \mu \mathrm{g}$ of the indicated scFv DNA vaccines or backbone vector. 4 days later, CD11 ${ }^{+}$ and CD11 $\mathrm{c}^{-}$cells were isolated and cocultured with CFSE-labeled OT-I T cells at the indicated APC to T cell ratios to determine CFSE dilution in vitro at day 7.5 of the experiment. (E) CD11c-DTR mice were vaccinated with $30 \mu \mathrm{g}$ scDEC-OVA or backbone vector and were depleted of CD11 $\mathrm{c}^{+}$DCs 4 days later by injection of $4 \mathrm{ng} / \mathrm{g}$ of DT (DT+); then CFSE-labeled OT-1 T cells were injected to monitor antigen presentation as in A. C-E are representative of 2 experiments.

To prove that MHC class I presentation by scDEC-OVA was DEC205 dependent, we performed similar experiments with DEC205-deficient (DEC-/-) mice and observed a lack of T cell proliferation (DEC ${ }^{-/-}$; Figure $\left.2 \mathrm{~B}\right)$. To prove that $\mathrm{MHC}$ class I presentation by the SCDEC-OVA was transporter for antigenic peptides-dependent (TAP-dependent), we verified that TAP-deficient (TAP ${ }^{-/-}$) mice could not induce OT-I T cell proliferation (Figure 2B).

To assess the duration of antigen presentation after scDEC-targeted DNA vaccination, B6 mice were immunized with $30 \mu \mathrm{g}$ DNA, and CFSE-labeled OT-I TCR transgenic T cells were transferred 7, 14,21 , and 28 days later. Antigen presentation was measured by CFSE dilution by the transferred $T$ cells in the draining inguinal as well as mesenteric and iliac lymph nodes and spleen. As shown in Figure 2C, presentation to OT-I T cells persisted for 4 weeks in the draining lymph nodes but decreased from week 1 to week 4 . Presentation in the mesenteric lymph nodes and spleen persisted for 2 weeks and declined rapidly at weeks 3 and 4. Injection of scControl-OVA resulted in only minimal presentation in the first week (Figure 2C). Together, the data in Figure 2, A-C, indicate that scDEC DNA vaccination improves the efficacy and duration of antigen presentation in vivo by a DEC-dependent mechanism.

To establish that scDEC-OVA DNA vaccination targets DCs in vivo, we first isolated CD $11 \mathrm{c}^{+}$DC-enriched and CD $11 \mathrm{c}^{-} \mathrm{DC}$ depleted cells from mice that were vaccinated with scDEC-OVA or scControl-OVA DNA. Then we cocultured the cells with CFSE-labeled OT-I TCR transgenic T cells as reporters for suc- 
A

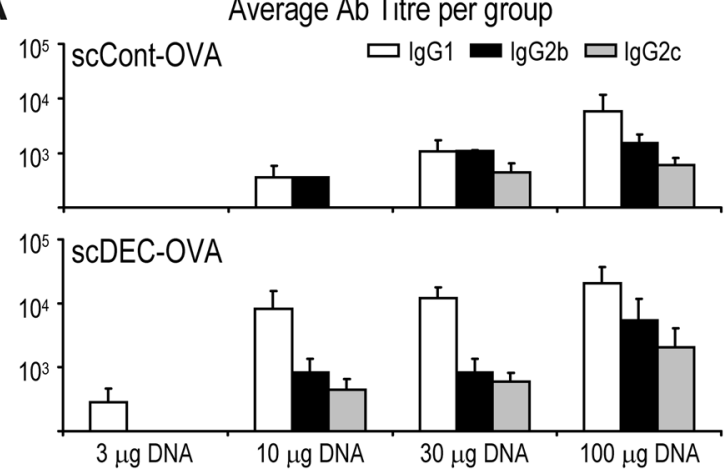

B

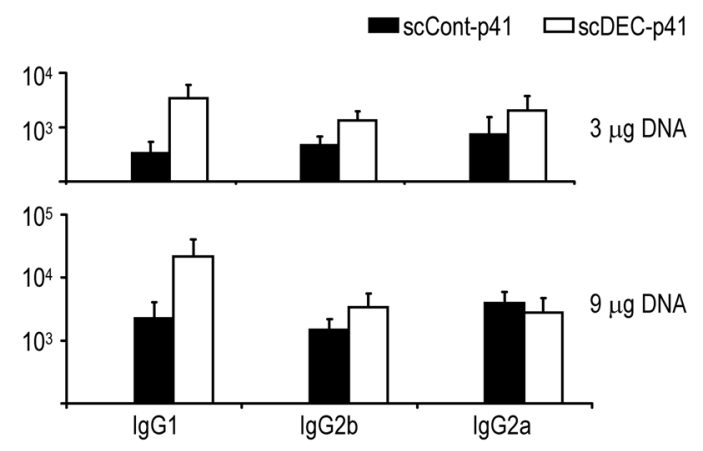

Figure 3

Targeted vaccination induces enhanced antibody responses. Antigen-specific antibody titers were determined for each mouse in a group $(n=5)$ from serum harvested at 3 or 5 weeks after vaccination with the indicated doses of DNA. (A) Titers of anti-OVA-specific isotype in B6 mice after $1 \mathrm{immunization}$ with graded doses of DNA vaccine i.m. in combination with electroporation. (B) Titers of anti-HIV gag p41 isotypes in BALB/C mice after 1 immunization with low doses of DNA vaccine i.m. in combination with electroporation. DNA vaccine type is displayed at the top. Antibody titers are expressed as group mean \pm SD. Results are representative of 3 experiments.

cessful antigen presentation. We could only detect presentation of the OVA peptide and consequent proliferation of the OT-I TCR transgenic T cells from cocultures with CD11 $\mathrm{c}^{+} \mathrm{DCs}$ and not with CD11 $\mathrm{c}^{-}$-presenting cells (Figure 2D). In addition, no proliferation was observed when we cocultured OT-I TCR transgenic T cells with CD11 $c^{+}$DCs from DEC205-deficient mice, indicating that DEC205 was necessary for the uptake of the targeted vaccine by CD $11 c^{+}$DCs (Figure 2D). Finally, CD $11 c^{+}$DCs isolated from mice treated with the control DNA vaccine only induced background levels of proliferation (Figure 2D). We conclude that the targeted DNA vaccine delivered antigen to CD $11 \mathrm{c}^{+}$ DCs in situ in a DEC205-dependent manner.

To investigate the role of DCs in targeted DNA vaccination in vivo, we used a transgenic mouse (CD11c-DTR/GFP) that allows for conditional ablation of $\mathrm{CD} 11 \mathrm{c}^{+} \mathrm{DCs}$ in vivo after administration of diphtheria toxin (DT) (30). These mice carry a transgene encoding a fusion protein of the DT receptor and GFP under control of the mouse CD11c promoter, allowing for depletion of DCs in vivo upon injection of DT. The CD11c-DTR/GFP mice as well as transgene-negative littermates and WT C57BL/6 mice were vaccinated with $30 \mu \mathrm{g}$ DNA i.m. and injected with DT on day 4 (4 ng/g body weight of DT i.p.). 24 hours later, the mice each received $3 \times 10^{6}$ CFSE-labeled TCR transgenic OT-1 T cells. Three days later, draining lymph node lymphocytes were evaluated for proliferation of the T cells. As shown in Figure 2E, temporal ablation of CD $11 \mathrm{c}^{+}$ DCs resulted in marked reduction in CFSE dilution. In contrast, WT littermates lacking the CD11c-DTR/GFP transgene and treated similarly with DT behaved like WT B6 mice, with virtually all the OT-I T cells going into cell cycle in mice following targeted DNA vaccination. Thus, the ablation of DCs hampered the ability of the targeted vaccine to induce OT-I T cell responses in vivo.

Targeted DNA vaccination improves antibody responses. To evaluate whether scDEC-OVA vaccination would improve B cell responses, we injected B6 mice with graded doses of the vaccine or scControl OVA and measured anti-OVA antibodies. Another control group received a dose of the backbone (empty) vector pcDNA3.1 equivalent to the highest dose of DNA vaccine (data not shown). At all doses of DNA, mice primed with scDEC-OVA DNA produced OVA-specific IgG1 antibody titers at least 10 times higher than those of groups primed with scControl OVA (Figure $3 \mathrm{~A}$ ). IgG2b and IgG2c titers were lower than IgG1, and the differences between scDEC-OVA and scControl-OVA were less than observed for IgG1 responses (Figure 3A).

We next tested to determine whether a single dose of scDEC-HIV gag p41 could induce better gag-specific antibody responses in $\mathrm{BALB} / \mathrm{c}$ mice (Figure $3 \mathrm{~B}$ ). Six 8-week-old mice were injected once with 3 and $9 \mu \mathrm{g}$ doses of DNA i.m. in combination with electroporation. Five weeks later, serum was tested for HIV gag p41-specific antibodies. Again, at least 10 -fold better antibody titers were detected after a single injection of just $3 \mu \mathrm{g}$ of the targeted vaccine, and the increase in titers was greatest for the IgG1 subclass of antibodies. Antibody responses due to the backbone vector (pcDNA3.1) alone were insignificant (data not shown). Thus, DEC205 targeting enhances the humoral responses to a DNA vaccine.

scDEC targeting improves HIV gag-specific T cell responses. To examine $\mathrm{T}$ cell immunity to HIV gag, we immunized mice with graded doses of scDEC gag p41 and control DNA vaccine and measured T cell responses with a library of 15 -mer "mimetope" peptide staggered every 4 amino acids along the HIV gag p41 sequence (26). Multiparametric intracellular cytokine assays showed that $\mathrm{C} \times \mathrm{B} 6$ $\mathrm{F} 1$ mice generated $\mathrm{T}$ cell responses after vaccination with low doses of the targeted vaccine ( 0.3 and $3 \mu \mathrm{g}$ DNA) whereas controls did not (Figure 4A). In contrast, at the higher doses that are typically used during DNA vaccine experiments in mice $(30 \mu \mathrm{g})$, both scControl and scDEC vaccines performed similarly in inducing IFN- $\gamma$-producing, gag-specific T cells. Similar findings were made with ELISPOT assays, including the fact that no IL-4- or IL-10-producing cells were detectable (data not shown).

T cells producing multiple cytokines appear to be especially effective in protective immunity (31-33). We therefore examined the quality of the $\mathrm{T}$ cell responses induced by DNA vaccination in terms of the capacity of gag-specific $\mathrm{T}$ cells to secrete multiple cytokines such as IFN- $\gamma$, TNF- $\alpha$, and IL-2. Fourteen days after immunization, scDEC-targeted vaccination preferentially induced gag-specific polyfunctional $\mathrm{CD}^{+} \mathrm{T}$ cells, including triple cytokine producers, at all doses of the vaccine tested (Figure 4B). In contrast, triple producing cells were not detected after 1 dose of the scControl DNA vaccine. More double cytokine-producing $\mathrm{CD}^{+} \mathrm{T}$ cells 
A $\underline{\text { day } 14}$

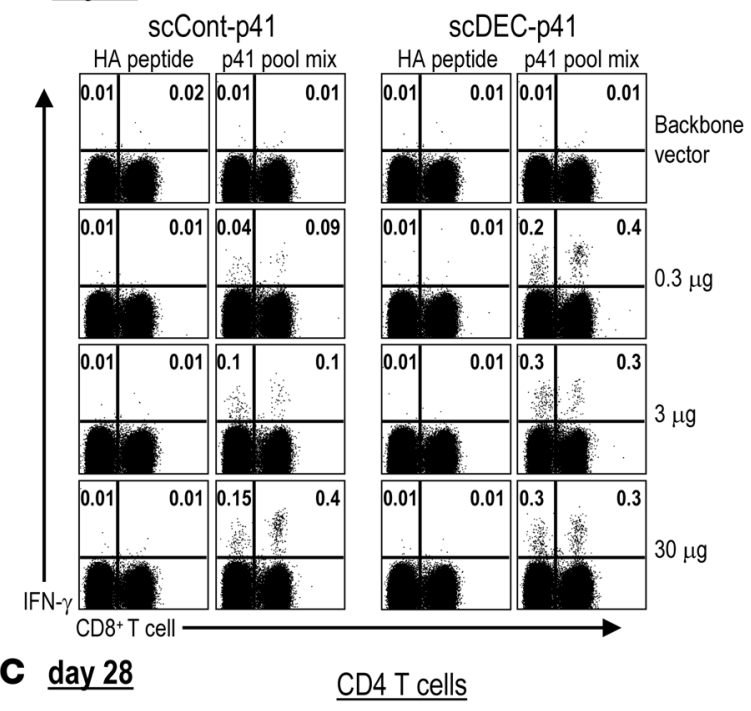

B $\underline{\text { day } 14}$

$\%$ Cytokine producing CD4 T cells

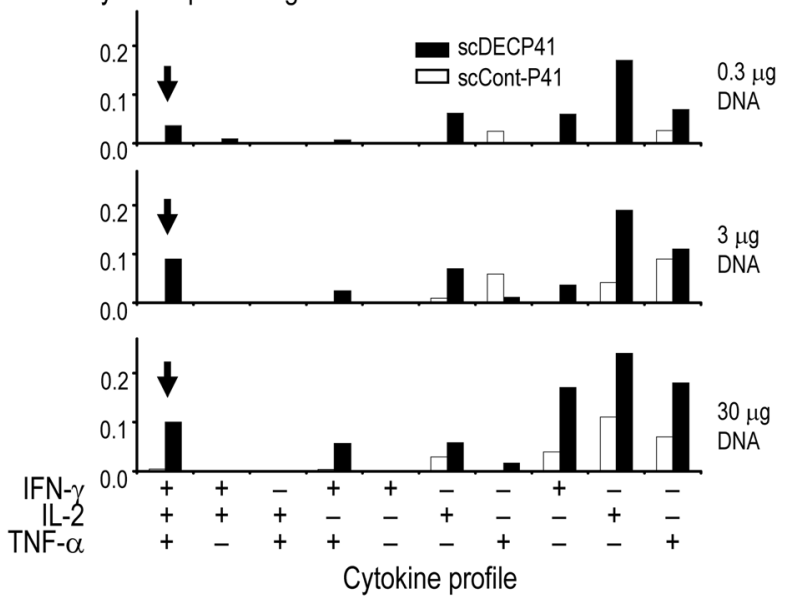

$\underline{\mathrm{CD} 8 \mathrm{~T} \text { cells }}$

$\%$ Cytokine producing CD4 T cells
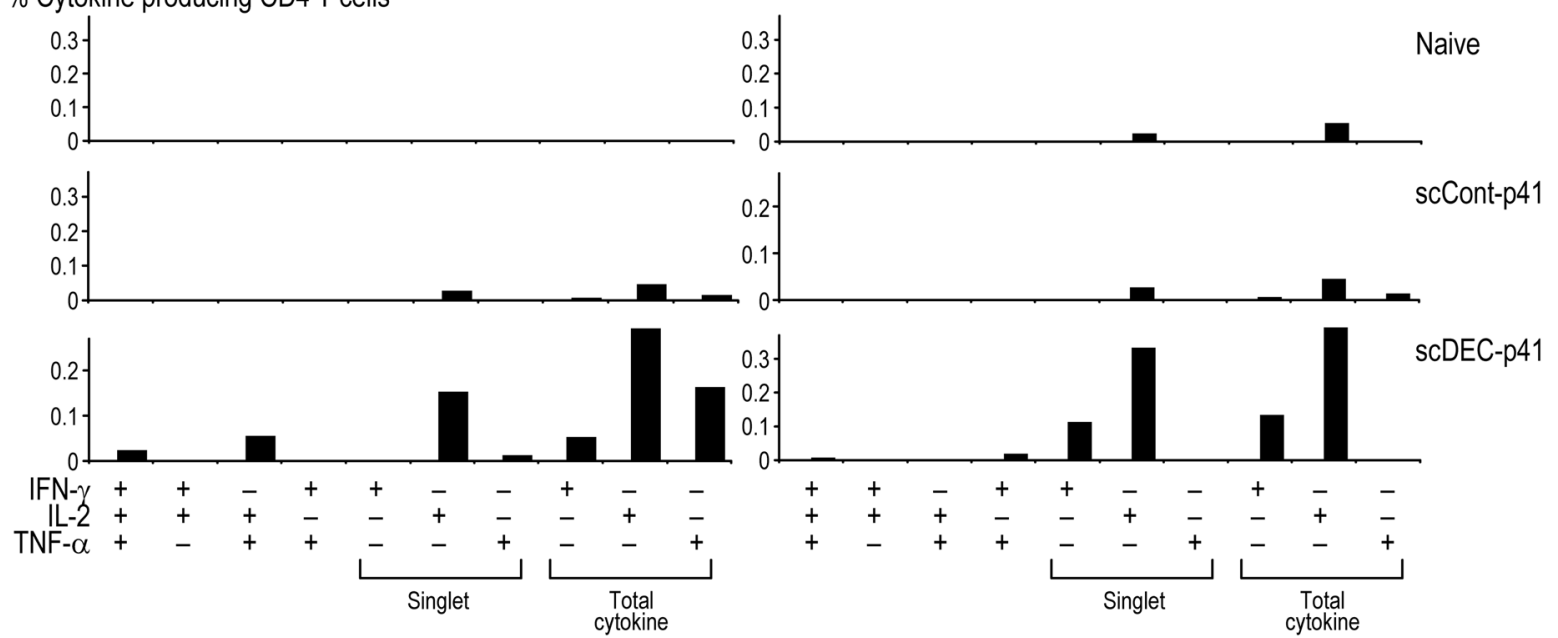

Cytokine profile

Figure 4

scDEC DNA vaccine improves HIV gag-specific T cell responses. Graded doses of the scDECp41 DNA vaccine alongside scControl DNA were administered i.m. in combination with electroporation to $\mathrm{C} \times \mathrm{B} 6 \mathrm{~F} 1 \mathrm{mice} .14$ days later, the percentage of IFN- $\gamma^{+} / \mathrm{TNF}-\alpha^{+} / \mathrm{IL}-2^{+}$T cells in gated CD3+ splenic $T$ cells was assessed using a gag p41 peptide pool mix. (A) IFN- $\gamma-$ secreting $C D 4^{+}$and $C D 8^{+} T$ cells among CD $3^{+} T$ cells in responses to unrelated peptide or HIV gag p41 peptide pool mix. (B) Cytokine profile of the primed CD4+ $\mathrm{T}$ cells 14 days after DNA vaccination. Cells secreting 3 (arrows), 2, or 1 cytokine are indicated on the $x$ axes. (C) Cytokine profile of the 3- $\mu$ g DNA vaccine dose 28 days after vaccination as in $\mathbf{B}$. Data are representative of 2 experiments with 2 mice pooled in each experiment.

were also induced at all doses of the scDEC vaccine (Figure 4B). The $\mathrm{CD} 4{ }^{+} \mathrm{T}$ cell responses to $\mathrm{scDEC}$ vaccine were broad since reactivity was noted to peptides in p24 pools 1 and 4 and the p17 pool (data not shown), as previously observed with anti-DEC antibody immunization (26). Responses to the backbone DNA vector were insignificant (data not shown). Likewise, at 28 days after vaccination (Figure 4C), scDEC-gag p41 induced cytokine-producing $\mathrm{CD}^{+}$and $\mathrm{CD}^{+} \mathrm{T}$ cells, including $\mathrm{T}$ cells with a predominant production of IL-2 as well as some double cytokine-producing T cells. These T cells expressed high levels of CD44, as is characteristic for memory $\mathrm{T}$ cells (not shown). These data indicate that scDEC targeting of low doses of DNA improves the induction of broad $\mathrm{T}$ cell-mediated immunity.
Targeted DNA vaccination elicits protection at a mucosal surface. To determine whether targeted vaccination produces long-lasting protective immunity at a mucosal surface, we challenged BALB/c and $\mathrm{C} \times \mathrm{B} 6 \mathrm{~F} 1$ mice intranasally with recombinant vaccinia-gag virus 3 months after a single dose of targeted and control vaccine, using a wide range of DNA doses, i.e., $0.3,3$, and $30 \mu \mathrm{g}$ per mouse. Control mice vaccinated with the empty vector were not protected relative to PBS-injected mice, and they lost weight and developed high lung virus titers over 7 days $\left(10^{7}-10^{8} \mathrm{PFU} / \mathrm{ml}\right.$ per lung; data not shown). In contrast, all mice receiving graded doses of the scDEC-targeted vaccine were protected, even with a single dose of $0.3 \mu \mathrm{g}$ DNA, whereas only mice vaccinated with the highest $30-\mu \mathrm{g}$ dose of the scControl vaccine showed protection (Figure 5A). 
A scCont-p41
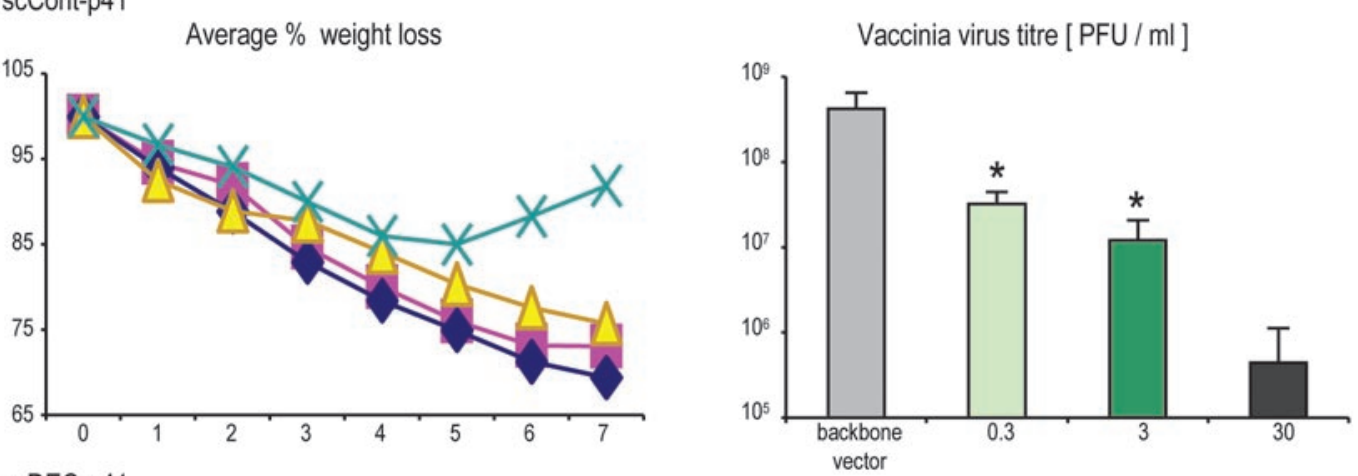

scDEC-p41 Average $\%$ weight loss

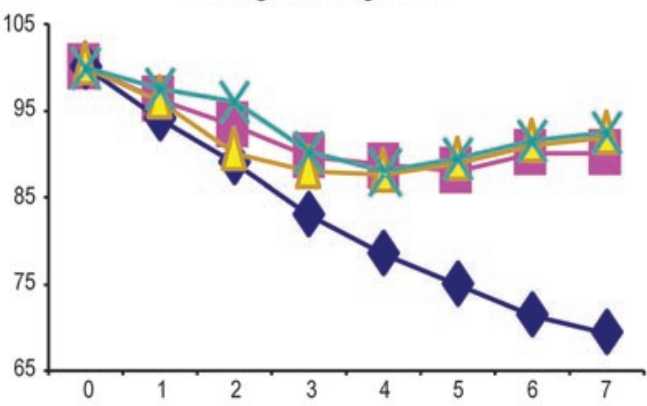

Vaccinia virus titre [PFU / ml ]
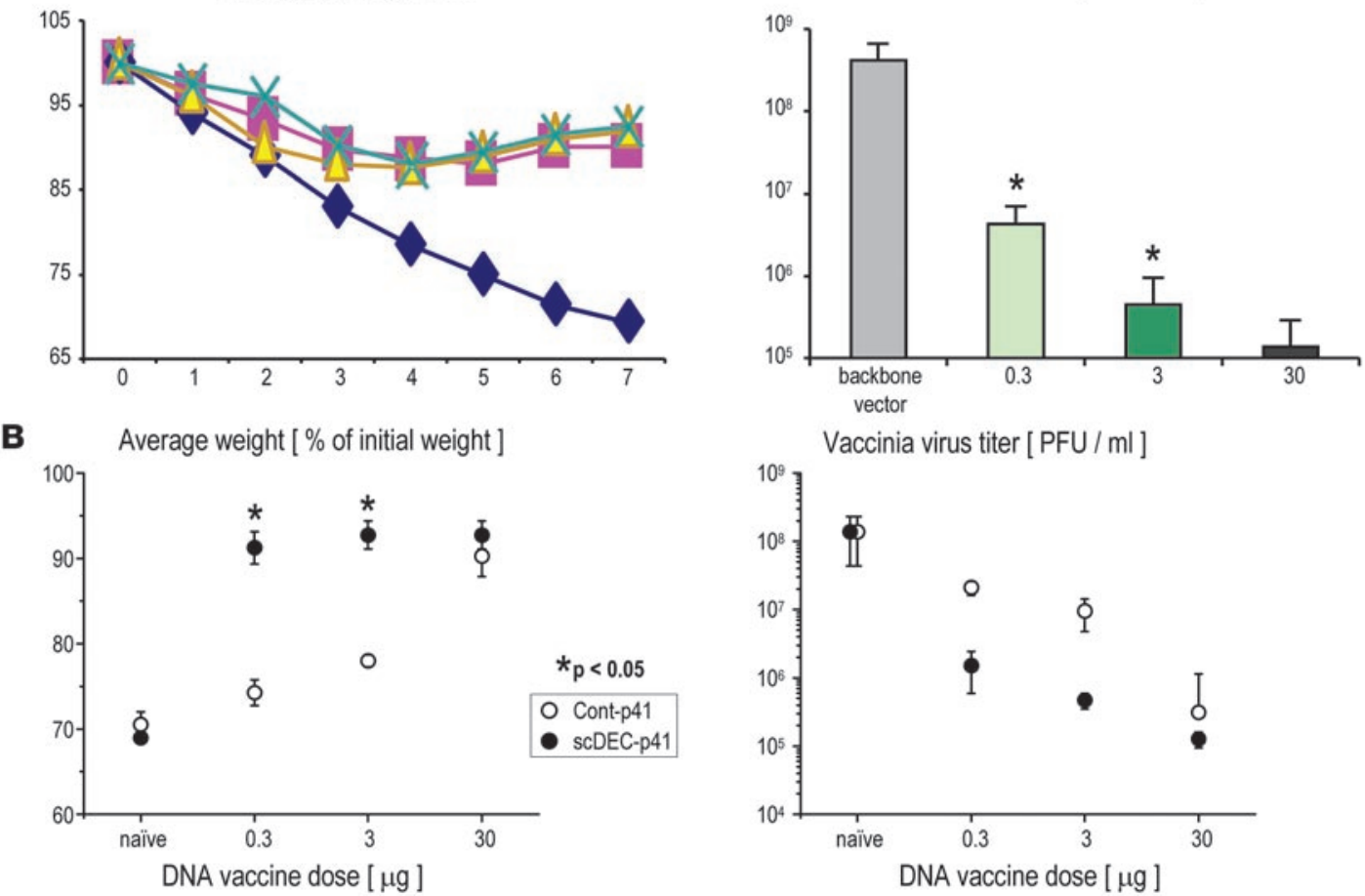

Figure 5

$A$ single, low-dose injection of DC-targeted DNA vaccine provides protective immunity to airway challenge with vaccinia gag virus. $\mathrm{C} \times \mathrm{B} 6 \mathrm{~F} 1 \mathrm{mice}$ received graded doses of ScDEC and scControl HIV gag DNA vaccine, and after 3 months, they were challenged i.n. with vaccinia gag virus. (A) Illustrative data of average weight (left panels) and mean \pm SD lung virus titers (right panels) after challenge. (B) Summary of data from a total of 4 different experiments (5 mice per group), 2 each in BALB/c and BALB/c $\times$ C57BI/6 F1 mice $\left({ }^{*} P<0.05\right)$.

Data from a total of 4 different experiments, 2 each in BALB/c and $\mathrm{BALB} / \mathrm{c} \times \mathrm{C} 57 \mathrm{Bl} / 6 \mathrm{~F} 1$ mice, are summarized in Figure $5 \mathrm{~B}$. It is evident that $\mathrm{scDEC}$ vaccine (Figure $5 \mathrm{~B}$ ) provides better and longlived protective immunity at lower $(0.3$ and $3 \mu \mathrm{g})$ but not higher $(30 \mu \mathrm{g})$ doses of DNA than the scControl (Figure 5B). Thus, the targeting of protein within a DNA vaccine to DCs in situ enhances protective efficacy by almost 100 -fold.

\section{Discussion}

Our results directly show that DNA vaccines can be configured to improve antigen presentation by DCs in vivo and that this in turn improves immunity and lowers the dose of DNA vaccine that is required. Multiple strategies have been developed to augment immune stimulation following DNA vaccination (reviewed in refs. 34-36). One approach is to include plasmids that encode different types of immunostimulatory molecules, such as cytokines (IL-2, IL-12, GM-CSF, flt-3L), chemokines (MIP-1 $\alpha$, SLC), and membrane costimulators (CD80, CD86) (37-43). Another approach is for the DNA vaccine to encode a fusion protein that allows the vaccine to target to specific immune molecules that could enhance immunization, e.g., MHC class II, CTLA-4, Fc receptors, and chemokine receptors (refs. 44-50; reviewed in ref. 6). A third, newer approach has been for the DNA vaccine to target pattern recognition and "alarmin" receptors to increase triggering of the innate immune system, e.g., DEC205/CD205, TLR5, and HSP70 $(27,51,52)$. This extensive literature has repeatedly shown that the immune response to DNA vaccines can be enhanced, but the findings have yet to 
prove that enhanced presentation of vaccine antigens by DCs is being achieved or that the dose of DNA required for vaccination can be greatly reduced.

To overcome these obstacles to improved DNA vaccines, we made plasmids in which the vaccine protein was fused to a singlechain antibody that could target the DEC205 receptor on DCs following secretion from transfected muscle. The rationale originated in prior research showing that hybrid antibodies carrying vaccine protein to target the DEC205 antigen uptake receptor on DCs could greatly increase antigen presentation and immune responses (24-26, 53-55). Here, we found that "hybrid" DNA vaccines, composed of scDEC205 or a single-chain Fv antibody (scFv) control Ig fused to another protein, were both secreted well from transfected cells. However, the introduction of an ScDEC205 antibody sequence into the DNA vaccine led to improved delivery of vaccine protein to $\mathrm{CD} 11 \mathrm{c}^{+} \mathrm{DCs}$ in situ relative to $\mathrm{scFv}$ control Ig and resulted in a large increase in the efficacy with which the vaccine induced $\mathrm{T}$ cell immunity to and protection against a challenge infection. Demangel et al. have previously reported on the use of scDEC205 to improve DNA vaccination (27). However, their study did not demonstrate targeting to DCs in lymphoid organs in a receptor-dependent manner, and they did not report a marked reduction in the dose of vaccine DNA that could elicit immunity, including protective immunity. Our data indicate that the scDEC vaccine greatly enhances, by about 100 -fold, the delivery of vaccine protein to DCs in vivo and allows for a corresponding reduction in the dose of DNA required to induce protective immunity.

Transfected muscle cells at sites of DNA injection may contribute to vaccine immunogenicity $(21,56)$. However, we feel that our results are best explained by the secretion of $\mathrm{scFv}$ anti-DEC vaccine from transfected muscle or other cells and then presentation by DCs targeted by the anti-DEC. This event is termed cross presentation because muscle cells synthesize the vaccine antigen, which then crosses into DCs for processing and presentation to $\mathrm{T}$ cells. Cross priming acts as a major mechanism for the induction of $\mathrm{T}$ cell responses following DNA vaccination $(16,17,19,20,22,23)$.

Hybrid $\alpha$ DEC antibody protein vaccines (24-26, 53-55) represent one strategy for preparing $\mathrm{DC}$-targeted vaccines, but the extension of this concept to DNA vaccines is limited by the need to coexpress the heavy and light chains in vivo to form a functional antibody within specialized antibody-producing B cells. The use of scFv $\alpha$ DEC-antigen fusion plasmids allows the antiDEC antibody to be secreted in tandem with the vaccine fusion protein from transfected non-B cells, and it allows for prolonged and greatly improved presentation of vaccine by DCs in lymphoid organs (Figures 1 and 2). In addition, we used an electroporation instrument to facilitate the delivery of DNA following i.m. injection, as has been reported by many others (e.g., refs. 29, 57-60). A newer finding is that electroporation may also make more DNA available for detection by intracellular DNA sensors, thereby activating the production of cytokines as an innate reaction (61). Thus, DNA electroporation in combination with DC targeting could provide a much more efficient platform for developing clinically effective DNA vaccines.

DCs are known to express several receptors with the potential to enhance antigen uptake (62). To date, the DEC205/CD205 receptor has been studied in the greatest detail in vivo and shown to deliver many different proteins for presentation on both MHC class I and II products $(24-26,53,55)$. DEC205 is expressed at high levels on a large fraction of DCs in the T cell areas of lymphoid organs, including human lymphoid tissues (15). However, other uptake receptors such as DCIR2 are preferentially expressed on different DC subsets with distinct antigen-presenting properties, and these too need to be assessed in single-chain antibody-based targeting vaccines (54). DEC205 targeting may be advantageous because it targets a subset of DCs in mice that is specialized to process antigens on MHC class I but also mediates improved immunization of MHC class II-restricted CD4+ helper T cells $(25,26,53)$.

Unlike protein-based DEC205 vaccines, which require the coinjection of additional DC maturation stimuli to induce $\mathrm{T}$ cell responses $(24-26,53,55)$, scDEC targeting in a DNA format induces immunity with no additional external adjuvant (27), possibly because the DNA itself provides some signals for DC maturation (61). In this study, we constructed several single-chain antibodyantigen fusion proteins that maintained the DEC205-binding specificity of the parental antibody. Injection of the scDEC DNA vaccine i.m. in combination with electroporation allowed antigen to be targeted to DCs in a DEC205-dependent fashion, resulting in a marked increase in presentation of antigen on MHC class I and II products. It needs to be determined whether the efficacy of scDEC-targeted vaccines can be further improved by the inclusion of additional stimuli that increase DC numbers and maturation state and/or improve the function of the responding $\mathrm{T}$ cells.

In summary, in vivo targeting of DNA-encoded vaccine proteins to DCs with scFv to DEC205 has the following features: (a) a precisely defined ratio of antigen to targeting antibody; (b) absence of binding via Fc receptors; (c) relative ease of large-scale production of DNA vaccine; and (d) increased immunogenicity (antibody as well as $\mathrm{CD}^{+}$and $\mathrm{CD}^{+} \mathrm{T}$ cells) because of the combination of DC targeting and stimulatory sequences within the vaccine. We propose that DC targeting via DEC205 or perhaps other DC receptors in the context of DNA vaccination will significantly enhance efficacy and may be particularly valuable for protection against diseases such as AIDS, in which broad-based immune responses will be helpful.

\section{Methods}

Vector construction. To generate scDEC-OVA plasmids, the cDNA of the heavy- and light-chain variable regions of the monoclonal antibody NLDC145 to DEC205 were amplified by PCR from the plasmids DECheavy HEL and DEC-K-HEL (24). Using overlap extension PCR with primers encoding an interchain linker (GGGGS) 6 TCCTCCGGAGGCGGTGGCTCAGGAGGTGGTGGGAGCGGTGGCGGCGGATCC), the heavy- and light-chain cDNAs were fused and cloned into the pcDNA3.1 expression plasmid (Invitrogen). A fragment containing the myc and histidine tags of plasmid VP22/myc-his (Invitrogen) was subsequently cloned downstream of the light chain. The OVA cDNA was amplified by PCR from plasmid pcDNA-OVA-L (63) and cloned in frame between the light chain and the tag, resulting in scDEC-OVA (Figure 1A). To generate scControl-OVA, heavy- and light-chain variable region cDNAs of the III/10 control antibody (which is the same isotype as the NLDC145 antibody but does not bind to DCs) (24) were also fused by overlap extension PCR and inserted in scDEC-OVA, replacing the respective DEC variable regions. Constructs were confirmed by sequence analysis. Similarly, HIV gag p24 and p41 were amplified from the plasmid syngag (64) and inserted in place of the OVA gene in the $\mathrm{scFv}$ vectors. For in vivo experiments, plasmid DNA was prepared using the QIAGEN Endo-Free plasmid kit.

Cell lines, media, and antibodies. 293 cells, 293 T cells (293ts/A1609), CHO cells, and CHOmDEC205 cells were maintained in DMEM supplemented with $10 \%$ FCS, penicillin, streptomycin, and glutamine. CV-1 cells were 
maintained in the same medium but with Geneticin in place of streptomycin. Antibodies specific for the TCRs of the transgenic OT-I and OT-II cells $\left(\mathrm{V} \beta_{5.1 / 5.2} / \mathrm{MR} 9-4 ; \mathrm{V \alpha}_{2} / 20.1\right)$ and to other cell surface markers (CD11c, $\mathrm{CD} 8 \alpha$, and CD4) were purchased from BD Biosciences - Pharmingen. Magnetic microbeads were from Miltenyi Biotec. Other antibodies included rabbit anti-OVA (Chemicon), FITC-labeled anti-p24 (KC57-FITC; Coulter) and HRP-conjugated monoclonal antibody to HIV gag p24 (ImmunoDiagnostics), and FITC-labeled goat anti-rabbit and goat anti-mouse polyclonal antibodies (BioSource).

SDS-PAGE and Western blot analysis. To assess production of scDEC and control antibody fusion protein, both the cell lysate and crude supernatants of the 293 transfectants were resolved on 10\% SDS-PAGE under reducing conditions and blotted onto nitrocellulose (Amersham) by standard techniques. The blots were blocked with $10 \%$ fat-free milk and then incubated with rabbit anti-OVA polyclonal antibody (1:10,000; Chemicon) followed by an anti-rabbit peroxidase conjugate $(1: 5,000)$. Bands were visualized with ECL substrate (Amersham). For the detection of HIV gag p24 and p41, the cell lysates and crude supernatants were similarly resolved on a 10\% SDS-PAGE and blotted with 1:7500 HRP-conjugated anti-p24 mAb.

$D N A$ vaccination. $\mathrm{BALB} / \mathrm{c}, \mathrm{C} \times \mathrm{B} 6 \mathrm{~F} 1$ mice were obtained either from Charles River, Taconic, or Harlan and maintained under specific-pathogen free conditions and used at 6 to 10 weeks under the guidelines of the Rockefeller University Institutional Animal Care and Use Committee. All experiments committee. Mice were injected with DNA i.m. in combination with electroporation in the anterior tibialis muscles of both hind legs. In brief, the mice were anesthetized with $1.25 \mathrm{mg} /$ mouse Nembutal. The skin overlying the anterior tibialis muscles of the hind legs was shaved and sterilized with ethanol swabs. DNA vaccine dissolved in $0.95 \% \mathrm{NaCl}$ was then injected i.m. followed immediately by electroporation using a 2-needle array electrode pair (Genetronics). The distance between the electrodes was $5 \mathrm{~cm}$, and the array was inserted parallel to the muscle fibers. 8 electric pulses of $200 \mathrm{~V} / \mathrm{cm}^{2}$ were delivered at 1-second intervals using a Bio-Rad gene pulser. Alternatively, DNA delivery and electroporation were achieved using an Ichor device (Ichor Medical Systems).

$T$ cell transfer. $\mathrm{CD}^{+}$or $\mathrm{CD}^{+} \mathrm{T}$ cells were prepared from spleen and lymph nodes of OT-I or OT-II mice, respectively, by negative selection using hybridoma supernatants directed against MHC-II, F4/80, B220, NK1.1, and CD4 or CD8 followed by goat anti-rat Dynabeads (Dynal). Purified OT-I and OT-II cells, $10^{7}$ cells $/ \mathrm{ml}$, were incubated with $5 \mu \mathrm{M}$ CFSE (Molecular Probes) for 10 minutes at $37^{\circ} \mathrm{C}$. An equal volume of FCS was added, and the cells were washed 2 times with PBS/0.1\% BSA and twice with PBS. $2 \times 10^{6}$ labeled OT-I and $3 \times 10^{6}$ labeled OT-II cells were injected i.v. into B 6 recipients that had been vaccinated 4-5 days previously either with scDEC-OVA or scControl-OVA DNA vaccines. After 3 days, cell suspensions of draining lymph nodes and spleen were stained for $\mathrm{V} \beta_{5.1 / 5.2}$ and CD8 (OT-I) or $V \alpha_{2}$ and CD4 (OT-II) using mAbs from BD Biosciences. The CFSE fluorescence intensity of OT-I and OT-II cells was then evaluated by multicolor flow cytometry using a FACScalibur (BD Biosciences) with subsequent analysis of data in CELLQuest (BD Biosciences) or FlowJo (Tree Star).

Assessment of DC targeting. C57BL/6(B6), TAP $-/$, DEC $-/$, OT-II, and OT-I mice were obtained from Charles River and maintained under standard conditions in the Rockefeller University animal facility. Four to five days after the injection of DNA vaccine, DCs were isolated from lymph nodes (popliteal, inguinal, axillary, brachial, mesenteric) and spleen. In brief, single-cell suspensions were prepared with $400 \mathrm{U} / \mathrm{ml}$ collagenase D (Roche) for 25 minutes at $37^{\circ} \mathrm{C}$. The cells were incubated with microbeads that had been coated with anti-mouse CD11c antibodies for 30 minutes at $4^{\circ} \mathrm{C}$. CD11 $\mathrm{c}^{+}$(DC-enriched) and CD11 $\mathrm{c}^{-}$cells were separated by application of a magnetic field. The isolated DCs were added in graded doses to the CFSElabeled OT-I cells $\left(0.2 \mu \mathrm{M}\right.$ CFSE $/ 10^{7}$ splenocytes for 10 minutes at $37^{\circ} \mathrm{C}$;
350,000 cells per well) at a DC:T cell ratio of 1:1, 1:3, and 1:9 in a final volume of $200 \mu \mathrm{l}$ RPMI 1640 culture medium containing 5\% FBS, $100 \mathrm{U} / \mathrm{ml}$ penicillin streptomycin mixture, $0.25 \mathrm{mg} / \mathrm{ml}$ Fungizone, $10 \mathrm{mM}$ HEPES, and $55 \mu \mathrm{M} \beta$-mercaptoethanol. Total cell numbers in each well were normalized by adding an appropriate number of DCs from naive mice. Three and one-half days later, OT-1 T cells were evaluated for proliferation by CFSE dilution. Similarly CD11c-DTR/GFP, transgene-negative littermates, or WT C57BL/ 6 mice were injected with $30 \mu \mathrm{g}$ of DNA i.m. in combination with electroporation. Five days later, the mice were injected i.p. with $4 \mathrm{ng} / \mathrm{g}$ body weight of DT, and 24 hours later, the mice each received $3 \times 10^{6}$ CFSE-labeled TCR transgenic OT-1 T cells. Three days later, draining lymph node lymphocytes were evaluated for proliferation of the T cells.

Serum antibody titers. For the detection of HIV gag p41-specific antibodies, high protein-binding ELISA plates were coated overnight at $4^{\circ} \mathrm{C}$ with $5 \mu \mathrm{g} / \mathrm{ml}$ of gag p 41 protein in PBS. Plates were washed 3 times with PBSTween $20(0.02 \%)$ and blocked with PBS-BSA 1\% for 2 hours at room temperature. Serum antibody titers were then determined as described (53), with the titer being the last dilution showing an $\mathrm{OD}_{405}$ greater than 0.1 .

$T$ cell responses to $s c D N A$ vaccines. To determine the breadth of HIV gagspecific $\mathrm{T}$ cell responses, bulk splenocytes were restimulated in vitro with peptide pools spanning the entire gag $\mathrm{p} 41$ protein (26). For the HIV gagspecific IFN- $\gamma /$ IL-2 ELISPOT assays, ELISPOT plates (MAIPS; Millipore) were coated overnight either with anti-mouse IFN- $\gamma$ or IL- 2 capture antibodies (BD Biosciences - Pharmingen). Bulk splenocytes were then cultured for 18 hours at $37^{\circ} \mathrm{C}$ with $5 \% \mathrm{CO}_{2}$ in the presence of $2 \mu \mathrm{M}$ of $\mathrm{HIV}$ gag p41 peptide pools as previously described (26). Plates were developed with anti-IFN- $\gamma$ biotinylated antibody (BD Biosciences) and spots visualized with avidin-horseradish peroxidase (Vector Laboratories), followed by $\mathrm{DAB}$ as substrate (Invitrogen). Spots were counted in an ELISPOT reader (Autoimmun Diagnostika GmbH). For multiparametric cytokine assays, bulk splenocytes were restimulated either with an $\mathrm{H}-2 \mathrm{~K}^{\mathrm{d}}$-restricted peptide $(2 \mu \mathrm{g} / \mathrm{ml}$; AMQMLKETI, p24 197-205), the entire HIV gag p41 peptide pool mix, a negative control influenza HA peptide mix, or medium alone in the presence of $2 \mu \mathrm{g} / \mathrm{ml}$ of anti-CD28 (clone 37.51) for 6 hours, adding $10 \mu \mathrm{g} / \mathrm{ml}$ of Brefeldin A (Sigma-Aldrich) for the last 4 hours to accumulate intracellular cytokines. Cells were stained as described (26), but dead cells were excluded using live/dead fixable dead cell stain kit (Aqua LIVE/DEAD; Invitrogen). After blocking Fc $\gamma$ receptors, the cells were stained with antibodies CD3-Pacific Blue, CD8-FITC, CD4-percp, CD44-Alexa Fluor 750 (CD27-PE), and Aqua LIVE/DEAD stain for 20 minutes at $37^{\circ} \mathrm{C}$. Cells were washed, fixed (Cytofix/Cytoperm Plus; BD Biosciences), permeabilized with PermWash and stained with antibodies to IFN- $\gamma$ (Alexa Fluor 700), TNF- $\alpha$ (Cy7-PE), and IL-2 (APCs) (eBioscience) for 15 minutes at room temperature. We used a BD LSRII with data analysis in FlowJo (Tree Star Inc.).

Vaccinia gag protection assays. Nembutal-anesthetized mice were challenged with $2.5 \times 10^{4}$ (B6 mice not shown), $5 \times 10^{4}(\mathrm{BALB} / \mathrm{C})$, and $1 \times 10^{5}(\mathrm{C} \times \mathrm{B} 6 \mathrm{~F} 1)$ PFUs of infectious virus by the intranasal route, in $35 \mu \mathrm{l}$ in PBS with $\mathrm{Mg} / \mathrm{Ca}$. The weight of each animal (groups of 5) was determined daily for 7 days after challenge. Then the lungs were removed and homogenized in transport medium $\left(0.1 \%\right.$ gelatin in PBS) and stored in duplicates at $-80^{\circ} \mathrm{C}$ prior to virus titration. Lung virus titers of individual mice in each group were determined by plaque assay on monolayers of CV-1 cells as described (26).

Statistics. Postchallenge mean vaccinia lung virus titers and mean percentage changes in weight were compared between vaccination groups using onetailed Student's $t$ test. Differences were considered significant at $P<0.05$. Statistical analysis was performed using Prism 3 (GraphPad Software).

\section{Acknowledgments}

We thank A. Charalambous and P. Konig for their contributions to this work; H. Zebroski (Rockefeller University proteomics 
facility) for synthesizing peptides; B. Moltedo and C.B. Lopez (Department of Microbiology, Mount Sinai School of Medicine) for recombinant vaccinia gag virus; and J. Adams for help with the graphics. This work was supported by grants from the NIH (AI40045 and 40874), the European community DEC-VAC project (IP Nr.: 018685), and the Wilhelm-Sander-Foundation (2004.107.1).
Received for publication October 12, 2007, and accepted in revised form January 23, 2008.

Address correspondence to: Ralph M. Steinman, The Rockefeller University, 1230 York Avenue, New York, New York 10065, USA. Phone: (212) 327-8107; Fax: (212) 327-8875; E-mail: steinma@ mail.rockefeller.edu.
1. Donnelly, J.J., Wahren, B., and Liu, M.A. 2005. DNA vaccines: progress and challenges. J. Immunol. 175:633-639.

2. Douek, D.C., Kwong, P.D., and Nabel, G.J. 2006. The rational design of an AIDS vaccine. Cell. 124:677-681.

3. Gurunathan, S., Wu, C.Y., Freidag, B.L., and Seder, R.A. 2000. DNA vaccines: a key for inducing longterm cellular immunity. Curr. Opin. Immunol. 12:442-447.

4. Ulmer, J.B., Wahren, B., and Liu, M.A. 2006. Gene-based vaccines: recent technical and clinical advances. Trends Mol. Med. 12:216-222.

5. Hokey, D.A., and Weiner, D.B. 2006. DNA vaccines for HIV: challenges and opportunities. Springer Semin. Immunopathol. 28:267-279.

6. Liu, M.A., Wahren, B., and Karlsson Hedestam, G.B. 2006. DNA vaccines: recent developments and future possibilities. Hum. Gene Ther. 17:1051-1061.

7. Wolff, J.A., et al. 1990. Direct gene transfer into mouse muscle in vivo. Science. 247:1465-1468.

8. Casares, S., Inaba, K., Brumeanu, T.-D., Steinman, R.M., and Bona, C.A. 1997. Antigen presentation by dendritic cells following immunization with DNA encoding a class II-restricted viral epitope. J. Exp. Med. 186:1481-1486.

9. Porgador, A., et al. 1998. Predominant role for directly transfected dendritic cells in antigen presentation to $\mathrm{CD}^{+} \mathrm{T}$ cells after gene gun immunization. J. Exp. Med. 188:1075-1082.

10. Bot, A., Stan, A.C., Inaba, K., Steinman, R.M., and Bona, C. 2000. Dendritic cells at a DNA vaccination site express the encoded influenza nucleoprotein and prime MHC class I-restricted cytolytic lymphocytes upon adoptive transfer. Int. Immunol. 12:825-832.

11. Akbari, O., et al. 1999. DNA vaccination: transfection and activation of dendritic cells as key events for immunity. J. Exp. Med. 189:169-178.

12. Condon, C., Watkins, S.C., Celluzzi, C.M., Thompson, K., and Falo, L.D.J. 1996. DNA-based immunization by in vivo transfection of dendritic cells. Nat. Med. 2:1122-1128.

13. Wiendl, H., Hohlfeld, R., and Kieseier, B.C. 2005. Muscle-derived positive and negative regulators of the immune response. Curr. Opin. Rheumatol. 17:714-719.

14. Lindquist, R.L., et al. 2004. Visualizing dendritic cell networks in vivo. Nat. Immunol. 5:1243-1250.

15. Granelli-Piperno, A., et al. 2005. Dendritic cell-specific intercellular adhesion molecule 3-grabbing nonintegrin/CD209 is abundant on macrophages in the normal human lymph node and is not required for dendritic cell stimulation of the mixed leukocyte reaction. J. Immunol. 175:4265-4273.

16. Corr, M., Lee, D.J., Carson, D.A., and Tighe, H. 1996. Gene vaccination with naked plasmid DNA: mechanism of CTL priming. J. Exp. Med. 184:1555-1560.

17. Doe, B., Selby, M., Barnett, S., Baenziger, J., and Walker, C.M. 1996. Induction of cytotoxic T lymphocytes by intramuscular immunization with plasmid DNA is facilitated by bone marrow-derived cells. Proc. Natl. Acad. Sci. U. S. A. 93:8578-8583.

18. Ulmer, J.B., Deck, R.R., DeWitt, C.M., Donnelly, J.J., and Liu, M.A. 1996. Generation of MHC classI restricted $\mathrm{T}$ lymphocytes by expression of a viral protein in muscle cells: antigen presentation by non-muscle cells. Immunology. 89:59-67.

19. Fu, T.M., et al. 1997. Priming of cytotoxic T lymphocytes by DNA vaccines: requirement for professional antigen presenting cells and evidence for antigen transfer from myocytes. Mol. Med. 3:362-371.

20. Iwasaki, A., Torres, C.A.T., Ohashi, P.S., Robinson, H.L., and Barber, B.H. 1997. The dominant role of bone marrow derived cells in CTL induction following plasmid DNA immunization at different sites. J. Immunol. 159:11-14.

21. Klinman, D.M., Sechler, J.M.G., Conover, J., Gu, M., and Rosenberg, A.S. 1998. Contribution of cells at the site of DNA vaccination to the generation of antigen-specific immunity and memory. J. Immunol. 160:2388-2392.

22. Corr, M., von Damm, A., Lee, D.J., and Tighe, H. 1999. In vivo priming by DNA injection occurs predominantly by antigen transfer. J. Immunol. 163:4721-4727.

23. Cho,J.H., Youn,J.W., and Sung, Y.C. 2001. Cross-priming as a predominant mechanism for inducing $\mathrm{CD}^{+}$ $\mathrm{T}$ cell responses in gene gun DNA immunization. J. Immunol. 167:5549-5557.

24. Hawiger, D., et al. 2001. Dendritic cells induce peripheral $\mathrm{T}$ cell unresponsiveness under steady state conditions in vivo. J. Exp. Med. 194:769-780.

25. Bonifaz, L.C., et al. 2004. In vivo targeting of antigens to maturing dendritic cells via the DEC-205 receptor improves T cell vaccination. J. Exp. Med. 199:815-824

26. Trumpfheller, C., et al. 2006. Intensified and protective $\mathrm{CD}^{+} \mathrm{T}$ cell immunity at a mucosal surface after a single dose of anti-dendritic cell HIV gag fusion antibody vaccine. J. Exp. Med. 203:607-617.

27. Demangel, C., et al. 2005. Single chain antibody fragments for the selective targeting of antigens to dendritic cells. Mol. Immunol. 42:979-985.

28. Widera, G., et al. 2000. Increased DNA vaccine delivery and immunogenicity by electroporation in vivo. J. Immunol. 164:4635-4640.

29. Zucchelli, S., et al. 2000. Enhancing B- and T-cell immune response to a hepatitis $C$ virus E2 DNA vaccine by intramuscular electrical gene transfer. J. Virol. 74:11598-11607.

30. Jung, S., et al. 2002. In vivo depletion of CD $11 c^{+}$ dendritic cells abrogation priming of $\mathrm{CD}^{+} \mathrm{T}$ cells by exogenous cell-associated antigens. Immunity. 17:211-220.

31. Harari, A., et al. 2006. Functional signatures of protective antiviral T-cell immunity in human virus infections. Immunol. Rev. 211:236-254.

32. Precopio, M.L., et al. 2007. Immunization with vaccinia virus induces polyfunctional and phenotypically distinctive $\mathrm{CD8}^{+} \mathrm{T}$ cell responses. J. Exp. Med. 204:1405-1416.

33. Darrah, P.A., et al. 2007. Multifunctional $\mathrm{T}_{\mathrm{H}} 1$ cells define a correlate of vaccine-mediated protection against Leishmania major. Nat. Med. 13:843-850.

34. Scheerlinck, J.Y. 2001. Genetic adjuvants for DNA vaccines. Vaccine. 19:2647-2656.

35. Barouch, D.H., Letvin, N.L., and Seder, R.A. 2004 The role of cytokine DNAs as vaccine adjuvants for optimizing cellular immune responses. Immunol. Rev. 202:266-274.

36. Kutzler, M.A., and Weiner, D.B. 2004. Developing DNA vaccines that call to dendritic cells. J. Clin. Invest. 114:1241-1244.
37. Iwasaki, A., Stiernholm, B.J.N., Chan, A.K., Berinstein, N.L., and Barber, B.H. 1997. Enhanced CTL responses mediated by plasmid DNA immunogens encoding costimulatory molecules and cytokines. J. Immunol. 158:4591-4601.

38. Barouch, D.H., et al. 2000. Control of viremia and prevention of clinical AIDS in rhesus monkeys by cytokine-augmented DNA vaccination. Science. 290:486-492.

39. Barouch, D.H., et al. 2002. Potent CD $4^{+}$T cell responses elicited by a bicistronic HIV-1 DNA vaccine expressing gp120 and GM-CSF. J. Immunol. 168:562-568.

40. Mwangi, W., et al. 2002. DNA-encoded fetal liver tyrosine kinase 3 ligand and granulocyte macrophage-colony-stimulating factor increase dendritic cell recruitment to the inoculation site and enhance antigen-specific $\mathrm{CD}^{+} \mathrm{T}$ cell responses induced by DNA vaccination of outbred animals. J. Immunol. 169:3837-3846.

41. Sumida, S.M., et al. 2004. Recruitment and expansion of dendritic cells in vivo potentiate the immunogenicity of plasmid DNA vaccines. J. Clin. Invest. 114:1334-1342.

42. Qin, H., et al. 2006. Enhancement of antitumour immunity by a novel chemotactic antigen DNA vaccine encoding chemokines and multiepitopes of prostate-tumour-associated antigens. Immunology. 117:419-430.

43. Yen, H.H., and Scheerlinck, J.P. 2007. Co-delivery of plasmid-encoded cytokines modulates the immune response to a DNA vaccine delivered by in vivo electroporation. Vaccine. 25:2575-2582.

44. Boyle, J.S., Brady, J.L., and Lew, A.M. 1998. Enhanced responses to a DNA vaccine encoding a fusion antigen that is directed to sites of immune induction. Nature. 392:408-411.

45. Deliyannis, G., Boyle, J.S., Brady, J.L., Brown, L.E., and Lew, A.M. 2000. A fusion DNA vaccine that targets antigen-presenting cells increases protection from viral challenge. Proc. Natl. Acad. Sci.U.S. A. 97:6676-6680.

46. You, Z., Huang, X., Hester, J., Toh, H.C., and Chen, S.Y. 2001. Targeting dendritic cells to enhance DNA vaccine potency. Cancer Res. 61:3704-3711.

47. Green, T.D., Montefiori, D.C., and Ross, T.M. 2003. Enhancement of antibodies to the human immunodeficiency virus type 1 envelope by using the molecular adjuvant C3d. J. Virol. 77:2046-2055.

48. de Arruda, L.B., Chikhlikar, P.R., August, J.T., and Marques, E.T. 2004. DNA vaccine encoding human immunodeficiency virus-1 Gag, targeted to the major histocompatibility complex II compartment by lysosomal-associated membrane protein, elicits enhanced long-term memory response. Immunology. 112:126-133.

49. Biragyn, A., Tani, K., Grimm, M.C., Weeks, S., and Kwak, L.W. 1999. Genetic fusion of chemokines to a self tumor antigen induces protective, T-cell dependent antitumor immunity. Nat. Biotechnol. 17:253-258.

50. Schiavo, R., et al. 2006. Chemokine receptor targeting efficiently directs antigens to MHC class I pathways and elicits antigen-specific CD8+ T-cell responses. Blood. 107:4597-4605.

51. Applequist, S.E., et al. 2005. Activation of innate immunity, inflammation, and potentiation of DNA vaccination through mammalian expression of the 
TLR5 agonist flagellin. J. Immunol. 175:3882-3891. 52. Hauser, H., and Chen, S.Y. 2003. Augmentation of DNA vaccine potency through secretory heat shock protein-mediated antigen targeting. Methods. 31:225-231.

53. Boscardin, S.B., et al. 2006. Antigen targeting to dendritic cells elicits long-lived $\mathrm{T}$ cell help for antibody responses. J. Exp. Med. 203:599-606.

54. Dudziak, D., et al. 2007. Differential antigen processing by dendritic cell subsets in vivo. Science. 315:107-111.

55. Soares, H., et al. 2007. A subset of dendritic cells induces $\mathrm{CD}^{+} \mathrm{T}$ cells to produce IFN- $\gamma$ by an IL-12-independent but CD70-dependent mechanism in vivo. J. Exp. Med. 204:1095-1106

56. Shirota, H., Petrenko, L., Hong, C., and Klinman,
D.M. 2007. Potential of transfected muscle cells to contribute to DNA vaccine immunogenicity. J. Immunol. 179:329-336.

57. Otten, G., et al. 2004. Enhancement of DNA vaccine potency in rhesus macaques by electroporation. Vaccine. 22:2489-2493.

58. Wu, C.J., Lee, S.C., Huang, H.W., and Tao, M.H. 2004. In vivo electroporation of skeletal muscles increases the efficacy of Japanese encephalitis virus DNA vaccine. Vaccine. 22:1457-1464.

59. Luxembourg, A., Hannaman, D., Ellefsen, B., Nakamura, G., and Bernard, R. 2006. Enhancement of immune responses to an HBV DNA vaccine by electroporation. Vaccine. 24:4490-4493.

60. Luckay, A., et al. 2007. Effect of plasmid DNA vaccine design and in vivo electroporation on the resulting vaccine-specific immune responses in rhesus macaques. J. Virol. 81:5257-5269.

61. Takaoka, A., et al. 2007. DAI (DLM-1/ZBP1) is a cytosolic DNA sensor and an activator of innate immune response. Nature. 448:501-505.

62. Figdor, C.G., van Kooyk, Y., and Adema, G.J. 2002. C-type lectin receptors on dendritic cells and Langerhans cells. Nat. Rev. Immunol. 2:77-84.

63. Diebold, S.S., Cotten, M., Koch, N., and Zenke, M. 2001. MHC class II presentation of endogenously expressed antigens by transfected dendritic cells. Gene Ther. 8:487-493.

64. Bojak, A., Wild, J., Wolf, H., and Wagner, R. 2002. Efficiency of a myogenic DNA vaccine is strictly dependent upon cellular localization of HIV-1 Pr55(gag). Vaccine. 20:1980-1984. 